\title{
Methods for grading severity of ocular involvement in patients with mucous membrane pemphigoid
}

\author{
Brendon W. H. Lee, 1,2,3 Melissa Radjenovic (MD), , ${ }^{1,2}$ Minas T. Coroneo (BSC MBBS MSC MD FRACS FRANZCO AO), ${ }^{1,3}$ \\ Dédée F. Murrell (MA BMBCh FAAD MD FACD) ${ }^{1,2}$

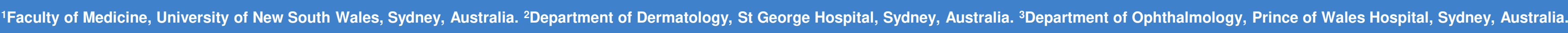

\section{Background}

- Mucous membrane pemphigoid with ocular involvement (OcMMP) is a bilateral sightthreatening disease, estimated to occur in $64-89 \%$ of MMP patients, usually between ages of 30-90 with female predominance. ${ }^{1}$

- Ocular involvement is thought to be due to the various biochemical and ultrastructural similarities common to the skin and cornea, which are both embryonically derived from the surface ectoderm. ${ }^{2}$

- OcMMP is characterised by ocular surface disease (OSD). Typically, cicatrising conjunctivitis with subepithelial fibrosis (Fig. 1) that progresses to symblephara formation, fornix foreshortening, corneal neovascularisation (Fig. 2), and ulceration.

- Scarring can also cause destruction of goblet cells, lacrimal gland ductules, and meibomian gland orifices leading to severe dry eye disease. ${ }^{3}$

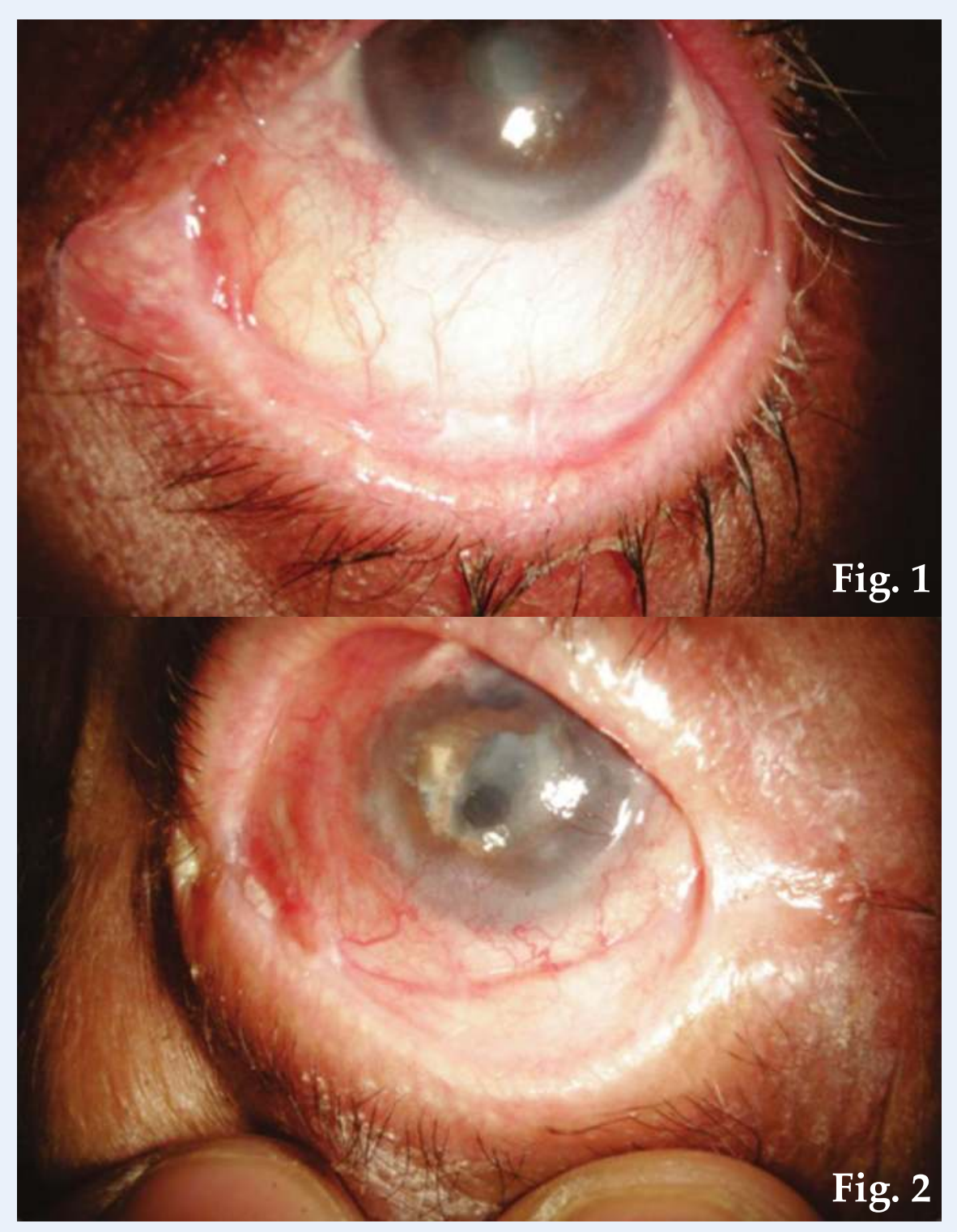

Materials \& Methods

The literature search was performed using three online databases: MEDLINE, EMBASE and Scopus. The following search terms were used: ['Pemphigoid, Benign Mucous

Membrane'] in different combinations with ['clinical assessment tool', 'conjunctiva', 'cornea', 'disease severity', 'disease scoring', 'eye disease', 'fornix', 'grading', 'ocular', 'progression', 'staging system']. Articles of al languages were included using translations.

\section{Results \& Discussion}

- Traditional methods focused on grading OcMMP severity using specific damagebased clinical parameters.

- Newer methods aimed to overcome identified difficulties by offering novel measuring techniques.

- Custom-designed fornix measurement tools have also been introduced (Fig. 3).

However, these tools focused on limited parameters i.e. disease activity was inferred purely from shifts between assessments.

- Significant variation would also occur when trying to measure the surface area of the conjunctiva's 3-D structure and when exposing the conjunctiva due to distortion, lid laxity, and severely fibrosed fornixes.
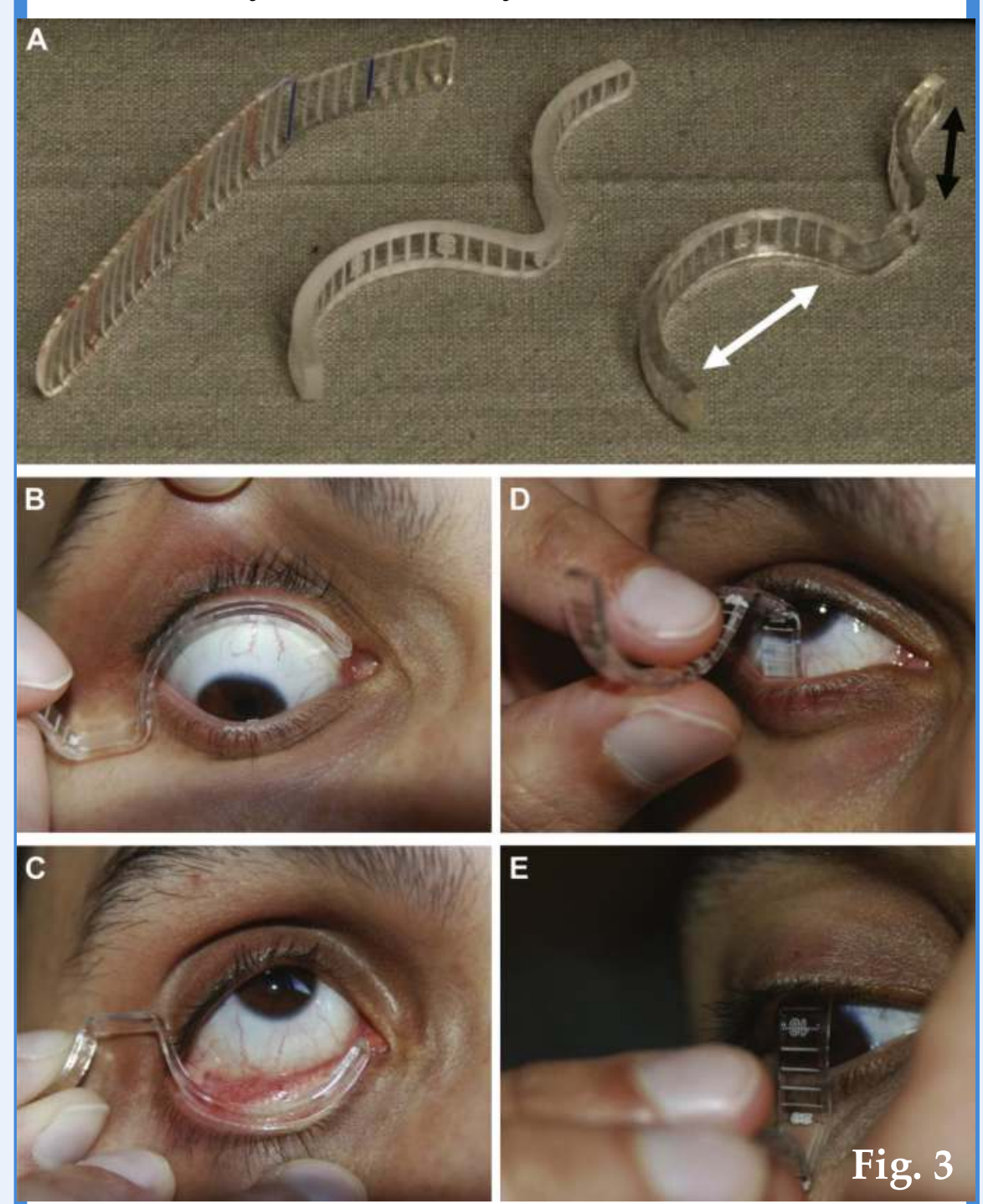

In 2017, international ophthalmic experts completed a Delphi Consultation and noted the incongruences of existing OSD scoring methods. ${ }^{4}$ They highlighted the importance of delineating clinical findings into 'activity' and 'damage' categories by creating the first universal 'toolbox' of parameters.

- This distinction can also be increasingly seen in the dermatological literature.

- Scoring activity as a separate entity, which can be reversed by treatment, would allow better appreciation of current severity and monitoring to prevent progression to permanent damage.

Table 1. Existing grading systems for OcMMP.
\begin{tabular}{l|l} 
Grade & Characteristics \\
\hline Foster (1986) \\
\hline I & Subconjunctival scarring and fibrosis. \\
\hline II & Fornix foreshortening (any degree). \\
\hline III & $\begin{array}{l}\text { Presence of symblepharon, and } \\
\text { degree. }\end{array}$ \\
\hline IV & Ankyloblepharon, frozen globe. \\
\hline
\end{tabular}

Mondino \& Brown (1987)

\begin{tabular}{l|l}
\hline I & $0-25 \%$ loss of inferior fornix depth
\end{tabular}

\begin{tabular}{l|l} 
II & $25-50 \%$ loss of inferior fornix depth
\end{tabular}

\begin{tabular}{l|l} 
III & $50-75 \%$ loss of inferior fornix depth \\
\hline
\end{tabular}

IV $\quad 75-100 \%$ loss of inferior fornix depth

Tauber (1992) - subdivisions of Foster II and III into a-d corresponding to Mondino and Brown.

\begin{tabular}{l|l}
\hline II a-d & $\%$ loss of inferior fornix depth
\end{tabular} III a-d $\%$ horizontal involvement of symblephara

Rowsey (2004) - total of 3 measurements of the distance from the inferior limbus to the posterior edge of the retracted lower eyelid $(5,6$, and 7 o' clock).

\begin{tabular}{l|l}
\hline $35-45 \mathrm{~mm}$ & $<25 \%$ conjunctival loss \\
\hline $24-34 \mathrm{~mm}$ & $25-50 \%$ conjunctival loss \\
\hline $12-23 \mathrm{~mm}$ & $50-75 \%$ conjunctival loss \\
\hline $0-11 \mathrm{~mm}$ & $>75 \%$ conjunctival loss
\end{tabular}

\section{Reeves (2012)}

Vertical grade: Distance from inferior limbus to start of fibrosis at 6 o' clock.

Horizontal grade: $\%$ involvement of bulbar conjunctiva width $2 \mathrm{~mm}$ above level of fibrosis, between the inner aspect of the nasal and lateral edges of the inferior posterior lid margin.

\begin{tabular}{l|l} 
a & $<25 \%$ conjunctival loss \\
\hline b & $25-50 \%$ conjunctival loss \\
\hline c
\end{tabular}

\begin{tabular}{l|l} 
c & $50-75 \%$ conjunctival loss
\end{tabular}

\begin{tabular}{l|l} 
d & $>75 \%$ conjunctival loss
\end{tabular}

Conclusions
- To the best of our knowledge, there is no
recognised comprehensive method of
staging and scoring the spectrum of
complications in OcMMP.
- Incorporating activity and damage into
future validated scoring tools would
facilitate more comprehensive assessments
and allow for evaluation of intervention
outcomes in clinical trials, longitudinal
monitoring of progression, and formulation
of treatment plans.
- With the multidisciplinary nature of care
for OcMMP patients, a standardised
method could provide much needed
commonality among clinicians.

○

\section{Literature Cited}

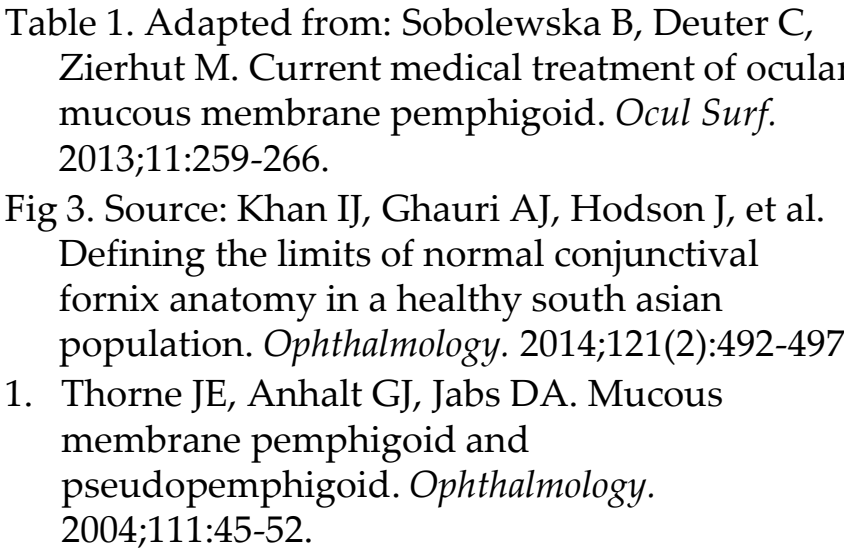

2. Figueira EC, Murrell DF, Coroneo MT.

Ophthalmic involvement in inherited 2010;28(1):143-152.

. Tan JCK, Murrell DF, Coroneo M. Ocular Involvement in Autoimmune Blistering Skin Diseases. In: Murrell DF, ed. Blistering Diseases: Clinical Features, Pathogenesis, Treatment. Berlin Heidelberg: Springer Berlin Heidelberg; 2015:449-455.

4. Mathewson PA, Williams GP, Watson SL, et al. Defining Ocular Surface Disease Activity and Damage Indices by an Internation Deyi

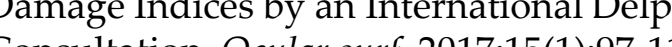
0

O

0

\section{Acknowledgements}

Thank you Dr. Revathi (DOS J: http://dx.doi.org/10.7869/djo.2013.7) for Fig 1. \& 2. Supported by the UNSW Independent Learning Program. Correspondence: please address to brendonlee@live.com.au \& d.murrell@unsw.edu.au. 